\title{
rAd5/NYVAC-B is superior to NYVAC-B/rAd5 and is dependent on rAd5 dose for neutralizing antibody responses against HIV-1
}

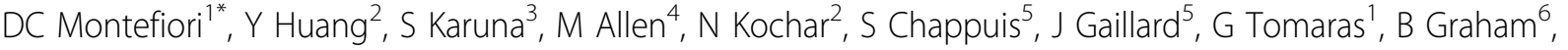 \\ P Bart ${ }^{5}, G$ Pantaleo ${ }^{5}$
}

From AIDS Vaccine 2012

Boston, MA, USA. 9-12 September 2012

\section{Background}

HVTN 078 is a phase $1 \mathrm{~b}$ clinical trial of heterologous vector prime/boost vaccine regimens (NYVAC-B/rAd5 vs. rAd5/NYVAC-B) in healthy, HIV-1 uninfected, Ad5 seronegative adults. The rAd5 expressed a clade B GagPol fusion protein and secreted gp140s of HIV-1 strains 92RW020 (clade A), HxB2/Bal-V3/ V1V2 (clade B) and 97ZA012 (clade C). The NYVAC-B expressed a clade B Gag-Pol-Nef polyprotein and the secreted gp120 of Bx08 (clade B). A total of 80 participants were randomized into a placebo group (P) and four treatment groups: T1, $2 x$ NYVAC-B $/ 1 x$ rAd5 $\left(10^{10}\right)$; T2, $1 x$ rAd5 $\left(10^{8}\right) / 2 x$ NYVAC-B; T3, $1 x$ rAd5 $\left(10^{9}\right) / 2 x$ NYVAC-B; T4, $1 x$ rAd5 $\left(10^{10}\right) / 2 x$ NYVAC-B.

\section{Methods}

Binding and neutralizing antibodies were assessed at 2 weeks post-final boosting. Neutralization was assessed with tier 1 and tier 2 Env-pseudotyped viruses in TZM-bl cells, and with tier 2 Env.IMC.LucR viruses in A3R5 cells.

\section{Results}

A dose effect for increasing anti-Env binding antibodies was seen, with higher doses of rAd5 being optimal. For neutralizing antibodies, positive response rates/median titers across the treatment groups were highest against MN.3 (69.3\%/116) followed by SF162.LS (42.1\%/54), BaL.26 (18.4\%/15.5), MW965.26 (14.5\%/31) and Bx08.16 $(11.8 \% / 19.5)$. Five subjects neutralized all 5 tier 1 viruses, 5 subjects neutralized 4 viruses, 7 subjects neutralized 3 viruses, 14 subjects neutralized 2 viruses (MN.3 and
SF162.LS) and 18 subjects neutralized 1 virus (MN.3). Aggregate magnitude-breadth scores across the tier 1 panel were strongest for $\mathrm{T} 4$ followed by $\mathrm{T} 3, \mathrm{~T} 1$ and $\mathrm{T} 2$. Differences were significant for T1 vs. T3 $(\mathrm{p}=0.048)$ and T1 vs. T4 $(\mathrm{p}=0.004)$. Responses against tier 2 viruses were weak and sporadic in the A3R5 assay and were nearly absent in the TZM-bl assay.

\section{Conclusion}

A $10^{10}$ dose of rAd5 was superior to the two lower doses of $10^{9}$ and $10^{8}$ for both binding and neutralizing antibodies. At the highest rAd5 dose tested, rAd5/ NYVAC-B was superior to NYVAC-B/rAd5 for neutralizing antibodies.

\section{Author details}

'Duke University Medical Center, Durham, NC, USA. ${ }^{2}$ SCHARP, Seattle, WA, USA. ${ }^{3}$ Fred Hutchinson Cancer Research Center/HVTN, Seattle, WA, USA. ${ }^{4}$ NIAID-NIH, Bethesda, MD, USA. ${ }^{5}$ Lausanne University Hospital, Switzerland. ${ }^{6}$ Vaccine Research Center, NIH, Bethesda, MD, USA.

\section{Published: 13 September 2012}

\section{doi:10.1186/1742-4690-9-S2-P132}

Cite this article as: Montefiori et al:: rAd5/NYVAC-B is superior to NYVAC-B/rAd5 and is dependent on rAd5 dose for neutralizing antibody responses against HIV-1. Retrovirology 2012 9(Suppl 2):P132.

${ }^{1}$ Duke University Medical Center, Durham, NC, USA

Full list of author information is available at the end of the article 